\title{
Percepción y conocimiento sobre COVID-19: Una caracterización a través de encuestas
}

\section{Perception and knowledge about COVID-19: A characterization through surveys}

Sebastian Iglesias-Osores ${ }^{1, a}$, Johnny Leandro Saavedra-Camacho, ${ }^{1, a}$, Johana Acosta-Quiroz ${ }^{1, a}$, Lizbeth M. Córdova-Rojas ${ }^{2, b}$, Arturo Rafael-Heredia ${ }^{3, c}$

\begin{abstract}
RESUMEN
Objetivo. Describir las percepciones y conocimientos sobre COVID-19. Material y Métodos: Estudio de corte transversal, se realizó una encuesta online a 314 participantes, el cuestionario consta de 9 preguntas de respuesta múltiple, los datos se analizaron mediante estadística descriptiva. Resultados: La edad promedio de los encuestados fue 26,9 años, la muestra comprendido más mujeres 161 (51,3\%) que hombres y casi 89\% de los encuestados pertenecían a pregrado, el lugar de origen del Covid-19 era bien conocido 94,30\%, para la mitad de los encuestados $159(50,6 \%)$ no se trata de una enfermedad zoonótica, con respecto a las medidas de prevención de COVID-19 la mayoría de los encuestados consideran que el lavado de manos es una de las principales medidas de prevención. Conclusión: Los estudios CAP son importantes para una mejor comprensión de la percepción y conocimiento de COVID-19. El estudio revelo que los encuestados generalmente tienen algún conocimiento general acerca de SARS-CoV-2.
\end{abstract}

Palabras Clave: Síndrome Respiratorio Agudo Grave; Betacoronavirus; Coronavirus; Infecciones por Coronavirus; COVID-19. Fuente: DeCS-BIREME).

\begin{abstract}
Objetive. To describe the perceptions and knowledge about COVID-19. Material and Method: Cross-sectional study, an online survey of 314 participants was conducted, the questionnaire consists of 9 multiplechoice questions, the data were analyzed using descriptive statistics. Results: The average age of the respondents was 26.9 years, the sample comprised more women $161(51.3 \%)$ than men and almost $89 \%$ of the respondents belonged to undergraduate, the place of origin of the Covid-19 was well known $94.30 \%$, for half of the respondents 159 (50.6\%) it is not a zoonotic disease, concerning the COVID-19 prevention measures the majority of the respondents consider that handwashing is an of the main prevention measures. Conclusion: CAP studies are important for a better understanding of the perception and knowledge of
\end{abstract}

Facultad de Ciencias Biológicas, Universidad Nacional Pedro Ruiz Gallo, Lambayeque, Perú. Universidad Nacional de Jaén, Cajamarca, Perú.

Facultad de Medicina Humana de la Universidad Nacional de Ucayali, Ucayali, Perú.

Biólogo.

Maestro en ciencias.

Doctor en ciencias de la salud.
COVID-19. The study revealed that respondents generally have some general knowledge about SARSCoV-2.

Keywords: Severe Acute Respiratory Syndrome; Betacoronavirus; Coronavirus; Coronavirus Infections; COVID-19 (Source: DeCS-BIREME).

\section{INTRODUCCIÓN}

Los coronavirus producen enfermedades del tracto respiratorio y gastrointestinal y tienen la capacidad de infectar a aves y mamíferos incluyendo los humanos ${ }^{(1)}$. Ya que son capaces de mutar recombinarse e infectar a distintas especies, aparentemente los murciélagos son un reservorio para estos virus ${ }^{(1)}$. Los coronavirus son virus de ARN que se dividen en cuatro tipos: alfa, beta, delta y gamma de los cuales CoV alfa y beta afectan a los humanos ${ }^{(2)}$.

A partir de diciembre de 2019 surgieron casos de una neumonía cuya etiología era desconocida en la ciudad de Wuhan en China ${ }^{(3)}$. La mayor parte de los pacientes habían visitado el mercado de mariscos de Wuhan, los científicos aislaron de un paciente un nuevo 
coronavirus, SARS-CoV-2 ${ }^{(4)}$.

Hay evidencia de que se transmite por contacto directo de persona a persona por lo tanto se deben se identificar medidas de control para aminorar la transmisión en la comunidad ${ }^{(5)}$. El brote de SARS CoV-2 se ha extendido por todo el mundo y se ha convertido en una emergencia de salud pública, esto ha llevado a la declaración de emergencia internacional por el brote de coronavirus $^{(6)}$. La población no tiene ninguna inmunidad inherente sobre este virus ${ }^{(7)}$.

Se conoce poco sobre sus manifestaciones clínicas $^{(3)}$. La mayoría de los pacientes de COVID-19 fueron diagnosticados con neumonía y presentaban patrones característicos en tomografía computarizada ${ }^{(8)}$. La prueba Gold Estándar para el diagnóstico de COVID-19 es la reacción en cadena de polimerasa con transcriptasa inversa (RT-qPCR) de muestras de vías respiratorias superiores o inferiores, sin embargo, esta técnica requiere un equipo especializado y puede tardar varias horas, recientemente se encuentran disponibles varias pruebas que detectan anticuerpos IgM, IgG contra el SARS CoV-2 que son rápidos y fáciles de usar, pero su utilidad clínica aun es controvertida ${ }^{(9)}$.

El virus ha infectado a un gran número de personas y la tasa de mortalidad es del $2 \%$ aproximadamente que es menor para el de otras epidemias por coronavirus como el Síndrome respiratorio agudo severo (SRAS) y síndrome respiratorio de medio oriente (MERS) ${ }^{(3)}$.

COVID-19 se ha convertido en un problema de salud pública que necesita un esfuerzo coordinado para combatirlo, se debe de fortalecer el monitoreo de SARS-CoV- $2^{(10)}$. Se debe determinar como el COVID-19 causa enfermedad y la repuesta inmunológica del huésped para poder encontrar una vacuna para disminuir y prevenir la enfermedad ${ }^{(1)}$. Los estudios CAP (conocimientos, actitudes y practicas) sobre Covid-19 son de mucha utilidad ya que los conocimientos son las bases para la prevención de la propagación de la enfermedad. El objetivo de este estudio es las percepciones y conocimientos sobre COVID-19.

\section{MATERIALY MÉTODOS}

\section{Población y diseño de estudio}

Estudio transversal utilizando una encuesta de cuestionario en línea a 314 personas llevado a cabo en Google formularios, la encuesta se compartió en redes sociales en los perfiles de los investigadores.

\section{Variables y mediciones}

Diseñamos un cuestionario online de 09 ítems que consiste en viñetas clínicas con preguntas de opción múltiple. Las preguntas en cada sección evaluaron el conocimiento de origen del nuevo coronavirus, vía de transmisión, letalidad, prevención, medios de comunicación, zoonosis, tratamiento y vacuna. Se realizó un estudio piloto en residentes de medicina interna $(n=37)$ y médicos $(n=6)$, que fueron excluidos del análisis posterior. Según los comentarios obtenidos, eliminamos 5 preguntas sobre medidas del gobierno, mejoramos la claridad de las preguntas. A los fines de este informe, describimos las respuestas a 9 preguntas de la encuesta. Luego publicamos este cuestionario utilizando un programa de encuestas en línea que permite al encuestado responder las preguntas y almacenar las respuestas en una hoja de Excel. El enlace a esta encuesta en línea, junto con una carta de presentación, se envió por redes sociales.

\section{Análisis estadístico}

Los datos se presentan como estadísticas descriptivas con tabulación. Las proporciones se calcularon en función del número total de encuestados para cada pregunta. El análisis estadístico se realizó con SPSS, versión 14.0 (SPSS Inc, Chicago, IL)

\section{Consideraciones éticas}

La participación fue voluntaria y las respuestas fueron anónimas. No se recopilaron datos de identificación. Las respuestas se recopilaron del 18 de febrero de 2020 al 01 de abril de 2020. Excluimos cuestionarios incompletos. Las respuestas de los residentes principales y los médicos asistentes se excluyeron del análisis. Se contó con el asentimiento de los encuestados y la aprobación del comité de ética de la Universidad Nacional Ucayali.

\section{RESULTADOS}

De acuerdo con los resultados obtenidos, el promedio de edad de los encuestados fue 26,90 años con un rango de edades entre los $16(1,30 \%)$ y $66(0,64 \%)$ años. El grado académico predominante de los encuestados fue pregrado $278(88,50 \%)$ frente a $36(11,50 \%)$ que fueron estudiantes de postgrado, la mayoría de los participantes pertenecen a carreras de ciencias de la salud $215(68,50 \%)$ y 99 (31,50\%) pertenecen a otras carreras. Respecto a la distribución según el sexo 161 $(51,30 \%)$ fueron mujeres y 153 (48,70 \%) fueron varones. (Tabla 1$)$.

Al indagar sobre los conocimientos acerca del SARSCoV-2 encontramos que 296 (94,30\%) sabe que el brote de este virus se originó en China mientras que 18 (5,70 $\%$ ) desconocen el lugar de origen del virus. De los 314 encuestados $224(71,30 \%)$ respondieron que la principal vía de transmisión es aerógena, en cuanto al nivel de letalidad $81(25,80 \%)$ de los encuestados piensan que tiene una letalidad baja, 103 (32,80\%) letalidad media y $130(41,40 \%)$ letalidad alta. En referencia a los conocimientos acerca si SARS-CoV-2 es una enfermedad zoonótica $155(49,4 \%)$ respondieron afirmativamente y para $159(50,60 \%)$ no la consideran una enfermedad zoonótica. Con respecto al 
conocimiento sobre el tratamiento del COVID-19 222 $(70,70 \%)$ opinan que existe tratamiento para la enfermedad y $92(29,30 \%)$ indicaron que no existe tratamiento. En cuanto a la pregunta sobre si se previene con una vacuna $194(61,80 \%)$ respondió que sí y $120(38,20 \%)$ respondió que no. La pregunta relacionada a si se trata del mismo coronavirus del perro y cerdo 174 (55,40\%) expreso que si mientras que $140(44,60 \%)$ de los encuestados opinan que no se trata del mismo.

Tabla 1. Distribución de los resultados de la encuesta sobre Covid-19

\begin{tabular}{|c|c|c|c|c|}
\hline & $\mathrm{n}$ & Media & D.E. & Porcentaje \\
\hline \multicolumn{5}{|l|}{ Grado } \\
\hline Pregrado & 278 & 156,68 & 90,49 & $88,50 \%$ \\
\hline Postgrado & 36 & 163,83 & 94,14 & $11,50 \%$ \\
\hline \multicolumn{5}{|l|}{ Profesión } \\
\hline Ciencias de la salud & 215 & 159,22 & 95,96 & $68,50 \%$ \\
\hline Otros & 99 & 153,76 & 78,71 & $31,50 \%$ \\
\hline \multicolumn{5}{|l|}{ Sexo } \\
\hline Femenino & 161 & 162,29 & 88,55 & $51,30 \%$ \\
\hline Masculino & 153 & 152.46 & 93,11 & $48,70 \%$ \\
\hline \multicolumn{5}{|l|}{ Origen del brote } \\
\hline China & 296 & 159,18 & 90,93 & $94,30 \%$ \\
\hline Otros & 18 & 129,89 & 86,14 & $5,70 \%$ \\
\hline \multicolumn{5}{|l|}{ Vía de transmisión } \\
\hline Aerógena & 224 & 152,10 & 91,03 & $71,30 \%$ \\
\hline Otros & 90 & 170,94 & 89,27 & $28,70 \%$ \\
\hline \multicolumn{5}{|l|}{ Letalidad } \\
\hline Baja & 81 & 194,53 & 81,21 & $25,80 \%$ \\
\hline Media & 103 & 151,98 & 93,07 & $32,80 \%$ \\
\hline Alta & 130 & 138,8 & 88,44 & $41,40 \%$ \\
\hline \multicolumn{5}{|l|}{$\begin{array}{l}\text { Es el COVID-19 una } \\
\text { zoonosis }\end{array}$} \\
\hline $\mathrm{Si}$ & 155 & 134,35 & 93,4 & $49,40 \%$ \\
\hline No & 159 & 180,07 & 82,39 & $50,60 \%$ \\
\hline \multicolumn{5}{|l|}{ Tratamiento } \\
\hline Sí & 222 & 157,24 & 91,91 & $70,70 \%$ \\
\hline No & 92 & 158,12 & 88,51 & $29,30 \%$ \\
\hline \multicolumn{5}{|c|}{ Se previene con vacuna } \\
\hline Sí & 194 & 158,55 & 90,73 & $61,80 \%$ \\
\hline No & 120 & 155,81 & 91,24 & $38,20 \%$ \\
\hline \multicolumn{5}{|l|}{ ¿Es el mismo } \\
\hline Sí & 174 & 161,91 & 94,19 & $55,40 \%$ \\
\hline No & 140 & 152,02 & 86,4 & $44,60 \%$ \\
\hline
\end{tabular}

Con respecto a las medidas de prevención de COVID-19 la mayoría de los encuestados consideran que el lavado de manos, cubrirse al toser y evitar el contacto con pacientes infectados son las principales medidas de prevención. (Figura 1) La pregunta acerca de los medios de comunicación solo la respondieron 121 encuestados donde predomina la radio y televisión 38 \% como el principal medio de comunicación donde se informaron sobre COVID-19. (Figura 2).

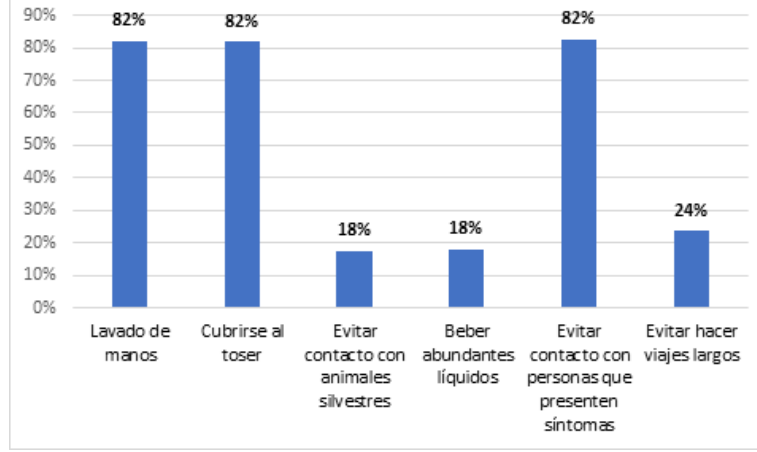

Figura 1.

Respuestas a ¿Qué opción cree usted que representa una medida de prevención contra la transmisión del coronavirus (2019-nCoV)? $(\mathrm{N}=314)$.

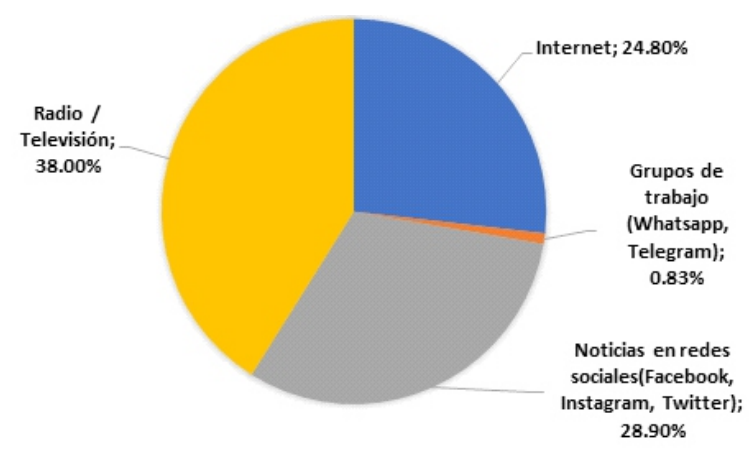

Figura 2.

Respuestas Medios de comunicación adquirió la información acerca del COVID-19 $(\mathrm{N}=121)$.

No se encontró correlación entre las variables de respuestas estudiadas y el grado de estudios, no se encontró correlación entre las variables de respuestas estudiadas y el tipo de profesión.

\section{DISCUSIÓN}

Se ha podido evidenciar que hay ciertas diferencias en cuanto a las respuestas de los individuos de nuestro estudio con los de otros países, como es el caso de los conocimientos acerca de las vías de transmisión de COVID-19 ya que en una investigación en China acertaron con la respuesta correcta (vía aerógena) cerca del $98 \%$ de los participantes ${ }^{(11)}$ al igual que afirmaron ciudadanos egipcios en un $96 \%^{(12)}$ y trabajadores de la salud en un hospital de Uganda en un $97 \%{ }^{(13)}$, en contraste con el $71,30 \%$ de nuestro estudio, con otro trabajo realizado en población también peruana que respondió bien en un $74 \%^{(14)}$, con una 
encuesta hecha en Italia hacia estudiantes de secundaria quienes respondieron correctamente cerca del $48 \%{ }^{(15)}$ y una investigación hecha en individuos estadounidenses (trabajadores de la salud y administrativos) que arrojó respuestas correctas en un rango de $55 \%$ a $75 \%{ }^{(16)}$, lo que indica un cierto desconocimiento o desinformación acerca de este tema en los individuos peruanos, norteamericanos e italianos. No obstante, para el enunciado acerca del origen chino del SARS-CoV-2, el estudio en individuos estadounidenses, respondió correctamente en más del $96 \%^{(16)}$, lo cual concuerda con nuestro estudio que llegó a casi un 94,3\%. Asimismo, las medidas de prevención, como se sabe, son imprescindibles para evitar la propagación del nuevo coronavirus, es por ello que, en el mismo estudio de China ${ }^{(11)}$, se acertó en casi un $99 \%$ que las personas deben reducir sus viajes en transportes públicos al igual que la investigación realizada en Uganda y Perú contestó correctamente en un $98 \%$ y $90 \%$ respectivamente en cuanto a la medida mencionada ${ }^{(13,14)}$, mientras que nuestro estudio, solamente un $23,6 \%$ consideró esta medida como importante, lo que sugiere, claramente que la población de China y Uganda consideran más preocupante el hecho de tener que tomar un transporte público que los individuos peruanos, de la misma manera, el lavado de manos y mantener distancia con personas sintomáticas es prioridad en los habitantes egipcios en casi un $100 \%^{(12)}$, para los trabajadores sanitarios en Uganda estuvieron de acuerdo con el lavado de manos antes y después de estar en contacto con personas sospechosas en un $88 \%^{(13)}$ igual que el $98 \%$ de ciudadanos peruanos en otro estudio(14); sin embargo, coincide con la encuesta italiana donde con respecto al lavado de manos, respondieron bien en un $75 \%$, sugiriendo que la mayoría de peruanos durante el periodo de pandemia, consideran estas dos últimas medidas como infaltables en su rutina diaria aunque otros usuarios no lo creen así. Es necesario recibir la más completa información acerca de la COVID-19 es por ellos que existen diversos medios de comunicación para ello. En la encuesta realizada hacia los estudiantes italianos, la mayoría recibieron información acerca de la enfermedad por televisión (56,3\%), seguida de sitios web en internet, diarios, familia, personal médico, redes sociales (Facebook, WhatsApp e Instagram) y sus respectivas escuelas, en forma descendente ${ }^{(15)}$; algo similar a nuestra investigación que en el mismo orden tenemos que la mayoría se informó acerca de dicha enfermedad por radio y televisión (38\%) seguida de redes sociales y sitios web en Internet, lo cual demuestra que los individuos elijen la radio y televisión como medio informativo principal, debido a distintas razones, una de ellas es la poca cobertura de internet en algunas zonas. Del mismo modo, el tratamiento es vital para combatir el COVID-19, es por ello que pobladores de Malasia afirmaron que, si había un tratamiento adecuado para esta enfermedad, si se trata con tiempo en un $94 \%^{\left({ }^{(17)}\right.}$, muy distinto de nuestra encuesta que solamente cerca del $30 \%$ afirmaron dicho enunciado, indicando falta de información. Finalmente, para los enunciados que tienen que ver con la tasa de letalidad, con el aislamiento social, con la infección zoonótica y vacunación con respecto a COVID19 , solamente se respondió de manera correcta la medida de la cuarentena para evitar la propagación del virus en un $98 \%$, para los otros enunciados aún se tiene un mayor desconocimiento acerca del tema, lo que sugiere a realizar mayores estudios y tratar de que el público en general conozca mejor a este nuevo patógeno ${ }^{(18-20)}$.

Este estudio es importante para una mejor comprensión de la percepción y conocimiento de COVID-19. El estudio revelo que los encuestados generalmente tienen algún conocimiento general acerca del COVID19 , sin embargo, aún hay algunos vacíos con respecto al nivel de letalidad, si se trata de una zoonosis o si es el mismo coronavirus que afecta al perro y cerdo, que necesitan más atención.

Conflictos de interés: Los autores niegan conflictos de interés.

Financiamiento: Autofinanciado.

\section{REFERENCIAS BIBLIOGRÁFICAS}

1. Fehr AR, Perlman S. Coronaviruses: An overview of their replication and pathogenesis. In: Coronaviruses: Methods and Protocols. Springer New York; 2015. p. 1-23.

2. Paules $\mathrm{Cl}$, Marston HD, Fauci AS. Coronavirus Infections-More Than Just the Common Cold. JAMA. 2020;323(8):707-8.

3. Chang D, Lin M, Wei L, Xie L, Zhu G, Dela Cruz CS, et al. Epidemiologic and Clinical Characteristics of Novel Coronavirus Infections Involving 13 Patients Outside Wuhan, China. JAMA. 2020;323(11):1092-3.

4. Kim JY, Choe PG, Oh Y, Oh KJ, Kim J, Park SJ, et al. The first case of 2019 novel coronavirus pneumonia imported into korea from wuhan, china: Implication for infection prevention and control measures. J Korean Med Sci. 2020;35(5):1-4.

5. Li Q, Guan X, Wu P, Wang X, Zhou L, Tong Y, et al. Early Transmission Dynamics in Wuhan, China, of Novel Coronavirus-Infected Pneumonia. N Engl J Med. 2020;

6. Habibzadeh P, Stoneman EK. The novel coronavirus: A bird's eye view. Int J Occup Environ Med. 2020;11(2):65-71.

7. Yee J, Unger L, Zadravecz F, Cariello P, Seibert A, Johnson MA, et al. Novel coronavirus 2019 (COVID-19): Emergence and implications for emergency care. J Am Coll Emerg Physicians Open. 2020;1:1-7. 
8. Zu ZY, Jiang M Di, Xu PP, Chen W, Ni QQ, Lu GM, et al. Coronavirus Disease 2019 (COVID-19): A Perspective from China. Radiology. 2020;

9. Imai K, Tabata S, Ikeda M, Noguchi S, Kitagawa Y, Matuoka $M$, et al. Clinical evaluation of an immunochromatographic IgM/lgG antibody assay and chest computed tomography for the diagnosis of COVID-19. J Clin Virol. 2020 Jul 1;128:104393.

10. Zhai P, Ding Y, Wu X, Long J, Zhong Y, Li Y. The epidemiology, diagnosis and treatment of COVID19. Int J Antimicrob Agents. 2020;

11. Zhong BL, Luo W, Li HM, Zhang QQ, Liu XG, Li WT, et al. Knowledge, attitudes, and practices towards COVID-19 among chinese residents during the rapid rise period of the COVID-19 outbreak: $A$ quick online cross-sectional survey. Int J Biol Sci. 2020;16(10):1745-52.

12. Abdelhafiz AS, Mohammed Z, Ibrahim ME, Ziady $\mathrm{HH}$, Alorabi M, Ayyad M, et al. Knowledge, Perceptions, and Attitude of Egyptians Towards the Novel Coronavirus Disease (COVID-19). J Community Health. 2020;

13. Olum R, Chekwech G, Wekha G, Nassozi DR, Bongomin F. Coronavirus Disease-2019: Knowledge, Attitude, and Practices of Health Care Workers at Makerere University Teaching Hospitals, Uganda. Front Public Heal. 2020;8(181).

14. Zegarra-Valdivia JA, Chino-Vilca BN, AmesGuerrero R. Knowledge, perception and attitudes in Regard to COVID-19 Pandemic in Peruvian Population. PsyArXiv. 2020;

15. Dilucca M, Souli D. Knowledge, attitude and practice of secondary school students toward COVID-19 epidemic in Italy: a cross selectional study. bioRxiv. 2020;
16. Modi PD, Nair G, Uppe A, Modi J, Tuppekar B, Gharpure AS, et al. COVID-19 Awareness Among Healthcare Students and Professionals in Mumbai Metropolitan Region: A Questionnaire-Based Survey. Cureus. 2020;12(4).

17. Azlan AA, Hamzah MR, Sern TJ, Ayub SH, Mohamad E. Public knowledge, attitudes and practices towards COVID-19: A cross-sectional study in Malaysia. medRxiv. 2020;

18. Wilder-Smith A, Freedman DO. Isolation, quarantine, social distancing and community containment: Pivotal role for old-style public health measures in the novel coronavirus (2019nCoV) outbreak. J Travel Med [Internet]. 2020 [cited 2020 May 24];27(2). Available from: https://pubmed.ncbi.nlm.nih.gov/32052841/

19. Kang L, Ma S, Chen M, Yang J, Wang Y, Li R, et al. Impact on mental health and perceptions of psychological care among medical and nursing staff in Wuhan during the 2019 novel coronavirus disease outbreak: A cross-sectional study. Brain Behav Immun. 2020 Mar;87:11-7.

20. Deng S-Q, Peng H-J. Characteristics of and Public Health Responses to the Coronavirus Disease 2019 Outbreak in China. J Clin Med. 2020 Feb 20;9(2):575.

\section{Correspondencia}

Sebastian Iglesias-Osores

Correo: sebasiglo@gmail.com

\section{Revisión de pares}

Recibido: 24/06/2020

Aceptado: 15/09/2020 